\title{
A multi-resource data integration approach: identification of candidate genes regulating cell proliferation during neocortical development
}

\author{
Cynthia M. Vied ${ }^{1+}$, Florian Freudenberg ${ }^{2+}$, Yuting Wang ${ }^{3+}$, Alexandre A. S. F. Raposo ${ }^{4+}$, David Feng ${ }^{5}$ \\ and Richard S. Nowakowski ${ }^{1 *}$
}

${ }^{1}$ Department of Biomedical Sciences, College of Medicine, Florida State University, Tallahassee, FL, USA

${ }^{2}$ Department of Psychiatry, Psychosomatics and Psychotherapy, University Hospital of Frankfurt, Frankfurt, Germany

${ }^{3}$ NUS Graduate School for Integrative Sciences and Engineering, National University of Singapore, Singapore, Singapore

${ }^{4}$ Molecular Neurobiology, Instituto Gulbenkian de Ciência, Oeiras, Portugal

${ }^{5}$ Allen Institute for Brain Science, Seattle, WA, USA

\section{Edited by:}

Rupert W. Overall, CRTD - Center for

Regenerative Therapies, Germany

Reviewed by:

Glenn D. Rosen, Beth Israel

Deaconess Medical Center/Harvard

Medical School, USA

Megan Kathleen Mulligan,

University of Tennessee Health

Science Center, USA

${ }^{*}$ Correspondence:

Richard S. Nowakowski,

Department of Biomedical Sciences,

College of Medicine, Florida State

University, 1115 West Call Street,

Tallahassee, FL 32306, USA

e-mail: richard.nowakowski@

med.fsu.edu

${ }^{\dagger}$ These authors have contributed equally to this work.
Neurons of the mammalian neocortex are produced by proliferating cells located in the ventricular zone (VZ) lining the lateral ventricles. This is a complex and sequential process, requiring precise control of cell cycle progression, fate commitment and differentiation. We have analyzed publicly available databases from mouse and human to identify candidate genes that are potentially involved in regulating early neocortical development and neurogenesis. We used a mouse in situ hybridization dataset (The Allen Institute for Brain Science) to identify 13 genes (Cdon, Celsr1, Dbi, E2f5, Eomes, Hmgn2, Neurog2, Notch1, Pcnt, Sox3, Ssrp1, Tead2, Tgif2) with high correlation of expression in the proliferating cells of the VZ of the neocortex at early stages of development (E15.5). We generated a similar human brain network using microarray and RNA-seq data (BrainSpan Atlas) and identified 407 genes with high expression in the developing human VZ and subventricular zone (SVZ) at 8-9 post-conception weeks. Seven of the human genes were also present in the mouse VZ network. The human and mouse networks were extended using available genetic and proteomic datasets through GeneMANIA. A gene ontology search of the mouse and human networks indicated that many of the genes are involved in the cell cycle, DNA replication, mitosis and transcriptional regulation. The reported involvement of Cdon, Celsr1, Dbi, Eomes, Neurog2, Notch1, Pcnt, Sox3, Tead2, and Tgif2 in neural development or diseases resulting from the disruption of neurogenesis validates these candidate genes. Taken together, our knowledge-based discovery method has validated the involvement of many genes already known to be involved in neocortical development and extended the potential number of genes by 100's, many of which are involved in functions related to cell proliferation but others of which are potential candidates for involvement in the regulation of neocortical development.

Keywords: the allen institute for brain science, neocortex development, gene expression, ventricular zone (VZ), subventricular zone (SVZ), GeneMANIA

\section{INTRODUCTION}

The neurons of the mammalian neocortex originate from neural progenitor cells (NPCs, either neuroepithelial cells or apical radial glia derived from the former) (Hartfuss et al., 2001; Kriegstein and Götz, 2003; Götz and Huttner, 2005). Initially, NPCs undergo symmetric proliferative divisions in the ventricular zone (VZ) of the dorsal telencephalon, amplifying their population. Cortical neurogenesis begins when these cells switch to differentiative asymmetric division, resulting in one daughter cell remaining an NPC and the other emerging as either a committed progenitor lineage or a postmitotic neuron (Götz and Huttner, 2005; Huttner and Kosodo, 2005). The intermediate progenitors move to the subventricular zone (SVZ), where they form an additional neurogenic layer that supplies projection neurons to all layers of the cerebral cortex (Takahashi et al., 1994,
1995, 1999; Haubensak et al., 2004; Kowalczyk et al., 2009). In addition to their role as progenitor cells, the bipolar apical radial glia at the VZ extend pial-directed processes which guide the migration of both intermediate progenitors and newly generated neurons (Ayala et al., 2007).

The VZ plays a central role in a complex and sequential process, requiring precise control of cell cycle progression, fate commitment, differentiation, and migration. Symmetric or asymmetric localization of subcellular components (Morin and Bellaïche, 2011), the Par complex (Wirtz-Peitz et al., 2008), signaling pathways including Notch (De la Pompa et al., 1997; Caviness et al., 2009), and the intercellular FGF and Wnt signaling pathways all interact with each other to regulate both proliferation and differentiation in the VZ of the neocortex (Johansson et al., 2010). 
The advent of whole genome technologies, particularly microarray and RNA-seq methods, has produced a wealth of information on the mammalian brain which has increasingly been deposited in publicly available databases. Consequently, we are now able to move from individual interactions to the identification of thousands of genes that are involved in biological processes. In this study, we used a knowledge-based discovery approach, analyzing publicly available databases from both mouse and human to identify candidate genes that are potentially involved in regulating early neocortical development and neurogenesis. Using this approach we have generated a network of 13 genes from mouse that are expressed in the VZ during early neocortical development. We also found over 400 candidate genes from human that are expressed in the VZ and SVZ at a comparable developmental time. As expected, many of these genes are known to be involved in the cell cycle, transcription and DNA replication. In addition, we used GeneMANIA and literature mining to generate extended mouse and human networks and to determine that at least two genes from the mouse network are potential novel regulators of neocortical development. This implies that some of the 400 human genes we identified could be novel regulators of early cortical development and could be the basis for future experimental studies.

\section{MATERIALS AND METHODS DIFFERENTIAL GENE EXPRESSION USING THE ALLEN DEVELOPING MOUSE BRAIN ATLAS}

To identify genes with high differential expression in the VZ of the developing mouse neocortex, we used the Allen Developing Mouse Brain Atlas (Thompson et al., 2014) $\left({ }^{(} 2013\right.$ Allen Institute for Brain Science. Allen Developing Mouse Brain Atlas. Available from: http://developingmouse.brain-map.org/). This atlas provides spatial expression patterns from in situ hybridization (ISH) images for 2104 genes related to brain development at multiple stages ranging between embryonic day 11.5 (E11.5) and postnatal day 56 (P56) of C57BL/6J mice. Specifically, we made use of the Allen Brain Atlas application programming interface (API) (http://www.brain-map.org/api/ index.html) to identify genes that are: (1) expressed in the telencephalon at E15.5, and (2) highly expressed in the VZ. The API's correlation search service yielded correlation scores for these genes. The most tightly correlated genes $(r>0.7)$ were used to create a Neocortex VZ Network. We analyzed expression of these genes in a qualitative manner by downloading high resolution ISH images for these genes from the Allen Developing Mouse Brain Atlas (http://help.brain-map. org/download/attachments/4325389/DevMouse_Overview.pdf).

In addition, analogous to the VZ network, we analyzed the Allen Mouse Brain Atlas for genes with specific expression in the neocortex outside of the VZ (Non-VZ Network; Figure S1). We did not further characterize the genes in the non-VZ network.

\section{IDENTIFICATION OF DIFFERENTIALLY EXPRESSED GENES USING THE BrainSpan ATLAS}

We identified genes differentially expressed in the VZ and SVZ of the human brain early in development using the BrainSpan
Atlas of the Developing Human Brain (Miller et al., 2014; ${ }^{\circ} 2014$ Allen Institute for Brain Science. BrainSpan Atlas of the Developing Human Brain. Available from: http://brainspan.org/). Specifically, we used the developmental transcriptome dataset, which is comprised of RNA sequencing and exon microarray data of $\sim 52,400$ genes from brains at different developmental stages [ 8 post-conceptual weeks ( $\mathrm{pcw}$ ) up to 39 years of age] to identify genes differentially expressed at $8-9$ pcw vs. all later stages. We used the prenatal laser microdissection (LMD) microarray dataset (comprised of microarray profiles from $\sim 58,700$ probes taken from $\sim 300$ different brain structures between 15 and $21 \mathrm{pcw}$ ) to find genes differentially expressed in the VZ and SVZ relative to the rest of the brain. For both datasets (developmental transcriptome and prenatal LMD microarray) we used the BrainSpan web interface to download fold-change and $p$-values for differentially expressed genes. Only those genes with at least six-fold higher expression (developmental transcriptome dataset: six-fold higher expression at 8-9 pcw vs. all later stages; LMD microarray dataset: six-fold higher expression in the VZ and SVZ compared to the rest of the brain) were used. Subsequently we corrected $p$-values using the Holm-Bonferroni method and set the significant $p$-value at $1 \times 10^{-9}$. We then compared the resulting lists for overlapping genes and used these genes for further analysis.

\section{GeneMANIA ASSOCIATION NETWORK}

GeneMANIA (http://genemania.org/) (Mostafavi et al., 2008) was used to find genes that are related to the 13 mouse VZ network genes (Figure 2A) and the 407 human network genes (Figure 5A). GeneMANIA uses functional association data including protein and genetic interactions, pathways, coexpression, co-localization, protein domain similarity and predicted interactions (based on organisms other than the one searched). GeneMANIA recommends using gene lists with no more than 100 genes for a search. Therefore, we used the top 100 genes from the human network plus the 7 mouse genes that overlapped between the mouse and human lists. All 13 mouse genes were searched through GeneMANIA. An additional 50 genes were shown in each network that are associated with the original $13 \mathrm{VZ}$ network genes or the 407 human network genes. The parameters used for the search, genes queried, associated gene lists, references used to generate the associations and all other outputs from GeneMANIA can be found in Tables S1,S4 for the mouse and human searches, respectively. The database version for these searches was June 1, 2014 and default settings were used for the appropriate organism for each list.

\section{GENE ONTOLOGY AND PATHWAY ENRICHMENT ANALYSIS}

WebGestalt, (http://bioinfo.vanderbilt.edu/webgestalt/; Zhang et al., 2005; Wang et al., 2013) and GOrilla (Eden et al., 2007, 2009), two web-based enrichment analysis platforms, were used to determine pathway and gene ontology (GO) enrichment. For pathway analysis in WebGestalt, a list of 407 genes correlated from the LMD microarray dataset and the developmental transcriptome was provided and the hsapiens_genome was used as the reference set to obtain significantly enriched [false discovery rate (FDR) adjusted $p$-value $<0.05$ ] pathways. Through WebGestalt we chose the Pathway Commons (Cerami et al., 2011) enrichment 
analysis. The statistical test used was hypergeometric and the Benjamini-Hochberg FDR method was used for multiple testing adjustment. GOrilla (http://cbl-gorilla.cs.technion.ac.il/) was used to identify GO terms that are significantly overrepresented in the list of 63 mouse extended network genes and 407 human SVZ and VZ genes. The mouse search was relative to a background set of the mouse genome and the human search was relative to approximately 18,000 human genes that could be associated with any GO term. GOrilla results were visualized as "TreeMaps" generated in REViGO (http://revigo.irb.hr/; Supek et al., 2011).

\section{LITERATURE MINING}

To validate the potential interaction among the $13 \mathrm{VZ}$ network genes, we performed a literature mining approach on published studies to generate a hypothetical functional gene network (Figure 6). A PubMed search was carried out using the keywords: (1) 13 gene symbols respectively; (2) combination of any two of the 13 gene symbols (e.g., Celsr1, Dbi). Specifically, "Cdo" and "Cdon" were used for Cdon studies, as "Cdo" was used as the gene symbol previously. Advanced search by "[Title/Abstract]" was performed for Notch1 because the number of articles was 3269 (indicated with “*”). Numbers of articles retrieved are listed in Table S6A. Titles, abstracts and "Materials and Methods" were examined to exclude non-mammalian or non-neuronal studies. Twenty-eight genes were identified that have interactions with the 13 candidates (Table S6B). The combination of any two of those genes were used as keywords in PubMed search (Table S6B). Advanced search by "[Title/Abstract]" was also used in some cases to control the number of studies (indicated with “*”). Non-mammalian or non-neuronal studies were excluded by scrutinizing titles, abstracts and "Materials and Methods." The hypothetical functional gene network contains the 13 candidates and a subset of the 28 genes that interacted with more than one gene found through the literature mining approach (Pani et al., 2002; Scardigli, 2003; Li et al., 2004, 2012a,b; Schuurmans et al., 2004; Yang et al., 2004; Taranova et al., 2006; Allen et al., 2007; Taylor et al., 2007; Nakazaki et al., 2008; Sawada et al., 2008; Shimizu et al., 2008; Shimojo et al., 2008; Wen et al., 2008; Yu et al., 2008; Favaro et al., 2009; Fernandez et al., 2009; Henke et al., 2009; Ochiai et al., 2009; Aguirre et al., 2010; Hu et al., 2010; Kaltezioti et al., 2010; Qu et al., 2010, 2013; Chavali et al., 2011; Gee et al., 2011; Karalay et al., 2011; SinorAnderson and Lillien, 2011; Taniguchi et al., 2012; Xia et al., 2012; Zhao et al., 2012, 2014; Zhang et al., 2012a,b, 2013; Imamura and Greer, 2013; Marqués-Torrejón et al., 2013; Petrova et al., 2013; Misra et al., 2014). Note that we listed only one article for each interaction (selection priority (original research articles only): in vivo > in vitro; direct interaction $>$ indirect interaction). However, for some correlations more than one article is accessible.

\section{RESULTS}

\section{MOUSE NEOCORTEX VENTRICULAR ZONE NETWORK}

To identify genes that are involved in neocortex development, we generated a network of genes that are differentially expressed in the VZ of the mouse embryonic neocortex (Figure 1A). We performed a correlation search for genes with similar spatial expression patterns using the API of the Allen Developing Mouse Brain Atlas from The Allen Institute for Brain Science (http:// developingmouse.brain-map.org/). This atlas has ISH data for over 2100 developmentally important genes during seven stages of development beginning at E11.5 through P56. We specifically analyzed data from E15.5, which is during the peak of neurogenesis for neocortex development. We graphed the most tightly correlated genes that are differentially expressed in the VZ of the neocortex at that time point (i.e., E15.5) compared to all other time points and brain areas (Figure 1). It should be noted that less than $10 \%$ of the protein-coding genes are represented in the Allen Developing Mouse Brain Atlas and therefore more genes could be expected in this network if all mouse genes were included.

We identified 13 genes in this search; Cdon, Celsr1, Dbi, E2f5, Eomes, Hmgn2, Neurog2, Notch1, Pcnt, Sox3, Ssrp1, Tead2, and Tgif2. We verified the expression and expression pattern of the 13 genes in the neocortex at E15.5 using the ISH data from the Developing Mouse Brain Atlas (Figures 1B-N). All 13 genes are predominantly expressed in the $\mathrm{VZ}$ of the neocortex. Three of those genes, Pcnt, Ssrp1, and Tead2 seem to be expressed in only a subset of the VZ cells. Pcnt (pericentrin) is expressed on the surface of the lateral ventricle (Figure 1J, arrowhead). This expression of Pcnt was observed previously (Miyoshi et al., 2006), however the functional consequences of this expression pattern are unknown. The same group reported PCNT protein localization at the base of primary cilia in E13 developing cerebral cortex, which is consistent with a role for Pcnt in microtubule organization (Miyoshi et al., 2006). The expression of Ssrp1 is higher in the outer half of the VZ (Figure 1L), and Tead2 is uniformly expressed in the VZ but not in all cells (Figure 1M). Cdon, Celsr1, Eomes, and Neurog2 (Figures 1B,C,F,H) are expressed more densely in the $\mathrm{VZ}$ of the neocortex than in the $\mathrm{VZ}$ of the ganglionic eminence (GE; arrow), suggesting that these genes serve a more specific role in neocortical VZ function.

Neurog2 is involved in generation of glutamatergic cortical neurons and also in repression of GABAergic neuron differentiation during cortical development (Roybon et al., 2010). Consistent with GABAergic neuron repression, Neurog2 expression is greatly reduced in the GE where GABAergic neurons originate (Figure $\mathbf{1 H}$, arrow). We found that a subset of genes in our VZ network (Figure 1A, box) also have reduced GE expression; Cdon, Celsr1 and Eomes (Figures 1B,C,F, arrow). All four genes have reduced GE expression at an earlier stage of development as well (E13.5, data not shown). Eomes is known to have a positive role in the migration of the GABAergic neurons from the GE to the SVZ of the neocortex (Sessa et al., 2010). Potentially, expression of Eomes in the GE could inhibit the migration of those neurons to the correct location in the SVZ. Cdon and Celsr1 are not known to be involved with GABAergic neuron migration, however, Celsr 1 has a positive role in the migration of facial branchiomotor neurons (Boutin et al., 2012). A role for Celsr1 and Cdon in GABAergic cortical neurons could be investigated based on the expression pattern similarity to Eomes and Neurog2 in the GE. 


\section{A Ventricular Zone Network}
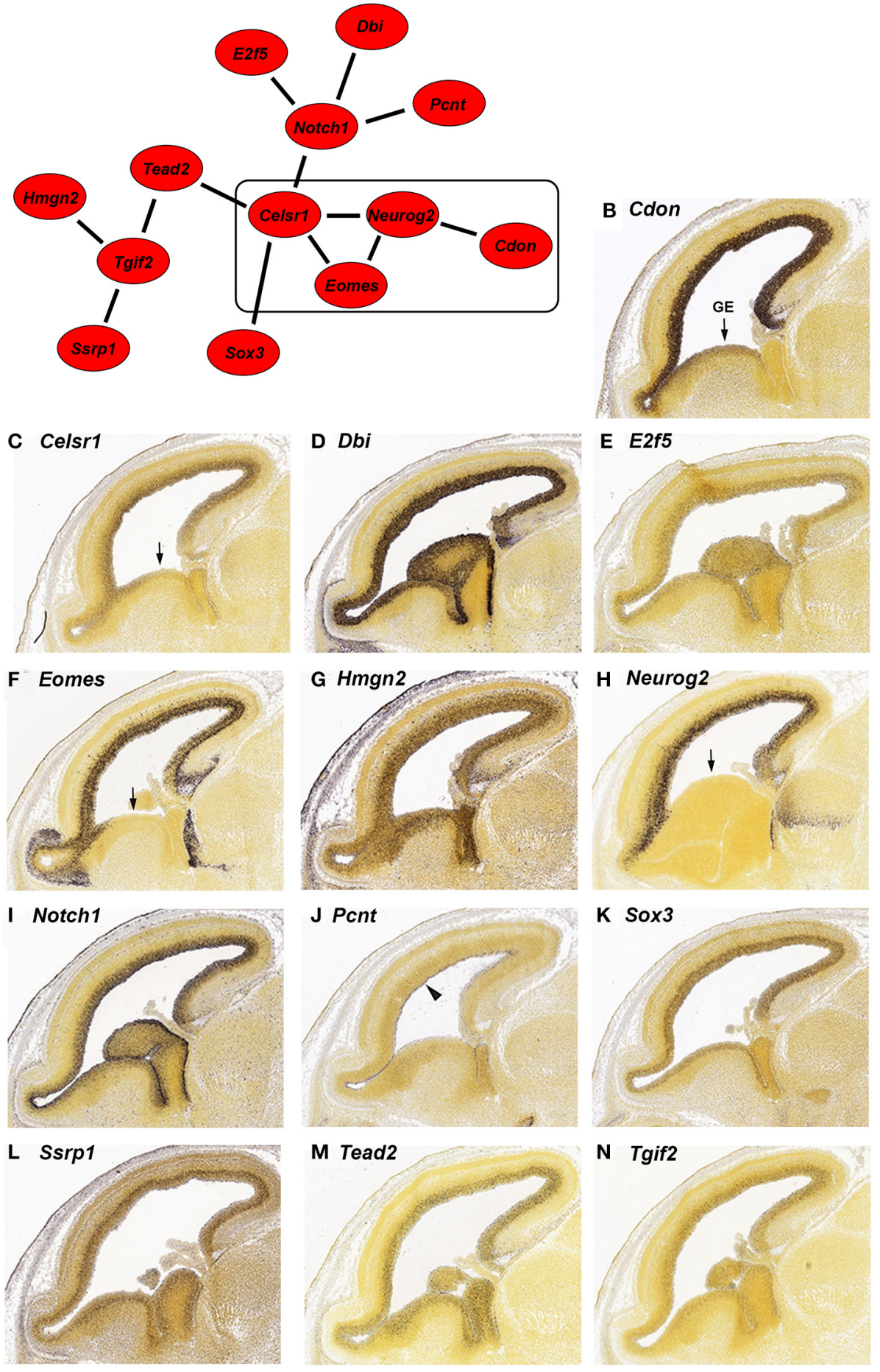

FIGURE 1 | In situ hybridization (ISH) of genes highly expressed in the ventricular zone (VZ) during embryonic development from the Allen Developing Mouse Brain Atlas. (A) Network of genes with high differential expression in the VZ of the mouse brain at age E15.5. The strength of correlation between the genes was at least an $r$-value of 0.7 . The rectangle indicates genes with reduced expression in the ganglionic eminence (GE). (B-N) Individual ISH images of genes from the VZ network (A). All genes show preferential expression in the proliferating cells of the VZ. Pcnt $\mathbf{( J )}$ is specifically expressed on the lateral wall of the lateral ventricle (indicated by the arrowhead). Four genes; Cdon (B), Celsr1 (C), Eomes (F), and Neurog2 (H) have decreased expression in the GE (indicated by arrows). 
In addition to $\mathrm{VZ}$ expression, we found that six of the $\mathrm{VZ}$ network genes (Dbi, Eomes, Hmgn2, Neurog2, Notch1 and Ssrp1; Figures 1D,F-I,L) are expressed at low to intermediate levels in regions of the neocortex outside of the VZ. Dbi (diazepam binding inhibitor, Figure 1D) is expressed in the VZ and SVZ. Consistent with a role for $D b i$ in the progenitor cells of the neocortex, $D b i$ has been shown to be involved in proliferation of SVZ progenitors in postnatal mice (Alfonso et al., 2012). Eomes (also known as Tbr2) and Neurog2 are additionally expressed in the SVZ and intermediate zone (IZ; Figures 1F,H). Both of these genes are transcription factors that regulate cortical progenitor cell maturation (Wilkinson et al., 2013; Sun and Hevner, 2014). Notch1, which is known to regulate multiple processes in the neocortex (Sun and Hevner, 2014), has reduced expression in the SVZ and moderate expression in the IZ (Figure 1I). Ssrp1 and Hmgn2 are chromatin binding proteins that have not been implicated in cortical development, although Ssrp1 has been shown to be important in Drosophila neural stem cell self-renewal (Neumüller et al., 2011). We found that both of these genes are highly expressed in the $\mathrm{VZ}$ and also show reduced expression in the other regions of the neocortex (Figures 1G,L). Taken together, these six genes are likely involved in multiple steps of cortical development, which has already been demonstrated for three of these genes; Eomes, Neurog2 and Notch1 (Sun and Hevner, 2014).

\section{GeneMANIA ASSOCIATION NETWORK AND GO ANALYSIS: MOUSE}

In order to predict potential interactions between the genes in our VZ network and with additional genes outside of the network, we generated an extended mouse network (Figure 2A). We used GeneMANIA (http://genemania.org/), a web-based interface that searches a large set of functional association data to return related genes based on available genomic and proteomic data. The association data include protein, DNA and genetic interactions, pathways, gene and protein expression data, phenotypic screens and shared protein domains (Zuberi et al., 2013). The VZ network gene list of 13 was extended to a list of 63 genes through GeneMANIA (Table S1 contains search parameters, the returned gene list, statistics used, plus additional outputs).

To predict the function of the 63 genes that are enriched in the extended mouse network, we examined GO enrichment of this gene list. We used publically available web-based GO analysis tools, GOrilla (http://cbl-gorilla.cs.technion.ac.il/) and REViGO (http://revigo.irb.hr/). We found a significant enrichment in genes involved in transcriptional regulation, anatomical structure development and macromolecule metabolism (Figure 2B and Table S2). We also evaluated GO enrichment in the original 13 genes from the mouse VZ network (data not shown). GO enrichment is not usually considered statistically significant in small gene lists, however, we did find that 10 of the 13 genes are involved in the regulation of transcription. This analysis demonstrates that the genes in our mouse $\mathrm{VZ}$ and extended networks are involved in transcriptional regulation, which is expected for genes implicated in early developmental processes such as neocortical development. In addition, the extended network is validated by common GO enrichment with the VZ network.

\section{HUMAN DEVELOPING BRAIN NETWORK}

To generalize the data from the mouse network we aimed to determine genes with high expression in the VZ and SVZ of the human neocortex early in development. To this end we made use of the BrainSpan Atlas of the developing human brain (http:// brainspan.org/). We started by identifying genes with strongest expression in the SVZ and VZ from the BrainSpan LMD microarray dataset (comprised of microarray profiles of samples taken from $\sim 300$ different brain structures at 15-21 pcw). Using the web interface of the BrainSpan atlas we identified data from 24,084 probes with at least the same level of expression (i.e., onefold) in the VZ and SVZ, compared to all other areas. From this list we removed all data points with less than a six-fold difference in expression in the VZ and SVZ compared to all other areas, resulting in a list of data points from 2746 remaining probes. After Holm-Bonferroni correction the $p$-values of all data points remained below the significant $p$-value of $1 \times 10^{-9}$. This set of 2746 data points contained 1781 different genes. Ten of the genes from this list (CDON, CELSR1, E2F5, EOMES, HMGN2, NEUROG2, NOTCH1, SOX3, TEAD2, TGIF2) were among the 13 mouse genes in the network described above (Figure 3).

In addition to identifying the genes with high expression in the SVZ and VZ, we wanted to identify those genes that are highly expressed early in development ( $\mathrm{pcw} 8-9$ ) compared to later time points. Again we employed the BrainSpan web interface to access the transcriptome dataset [comprised of RNA sequencing and exon microarray data from brains at different developmental stages ( 8 pcw up to 39 years of age)]. Our query resulted in a list of 18,105 genes with $=$ one-fold expression at pcw 8-9 compared to all later stages. This list was narrowed down to 1176 after removing all genes with less than a six-fold expression difference at pcw 8-9 compared to all later stages. After Holm-Bonferroni correction 1048 of these genes remained below the significant $p$-value of $1 \times 10^{-9}$. Eight of these genes (CELSR1, HMGN2, NEUROG2, NOTCH1, SOX3, SSRP1, TEAD2, TGIF2) were also present in the mouse network described above (Figure 4).

We then compared the resulting gene lists from the LMD microarray dataset and the developmental transcriptome, and found that 407 genes were overlapping between these two lists (Table S3). From these 407 genes, seven (CELSR1, HMGN2, NEUROG2, NOTCH1, SOX3, TEAD2, TGIF2) were also present in the mouse $\mathrm{VZ}$ network. An additional six genes overlap with the mouse extended network (FZD2, HIST1H3B, NDE1, SOX2, $T E A D 1$ and YAP1). The overlap between these networks is a further validation of the publically available datasets and methods used to generate the networks.

\section{GeneMANIA ASSOCIATION NETWORK: HUMAN}

To further evaluate the genes involved in human VZ and SVZ development, we generated an extended human network using GeneMANIA (Figure 5 and Table S4). Due to the length of the human gene list we used the top 100 human genes and the 7 genes that overlapped with the mouse VZ network to generate the extended network. As with the mouse extended network, we allowed 50 additional genes to be added to create our extended network. Interestingly, 46 of the 50 genes 
A

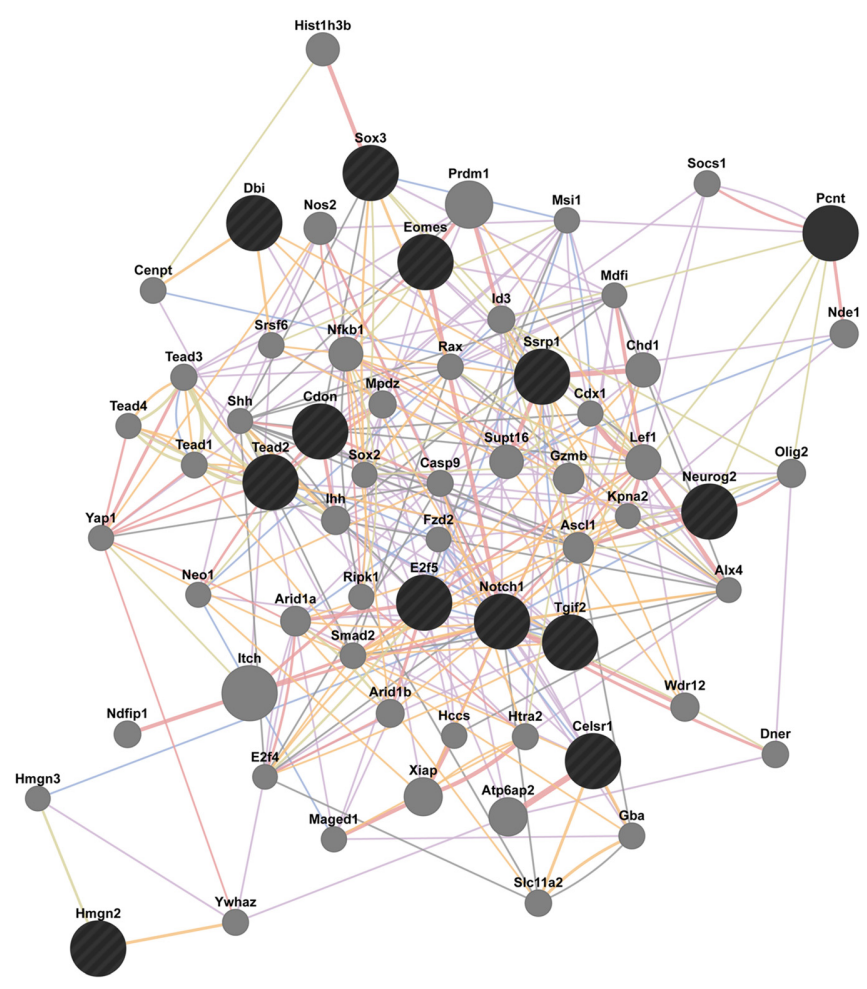

B

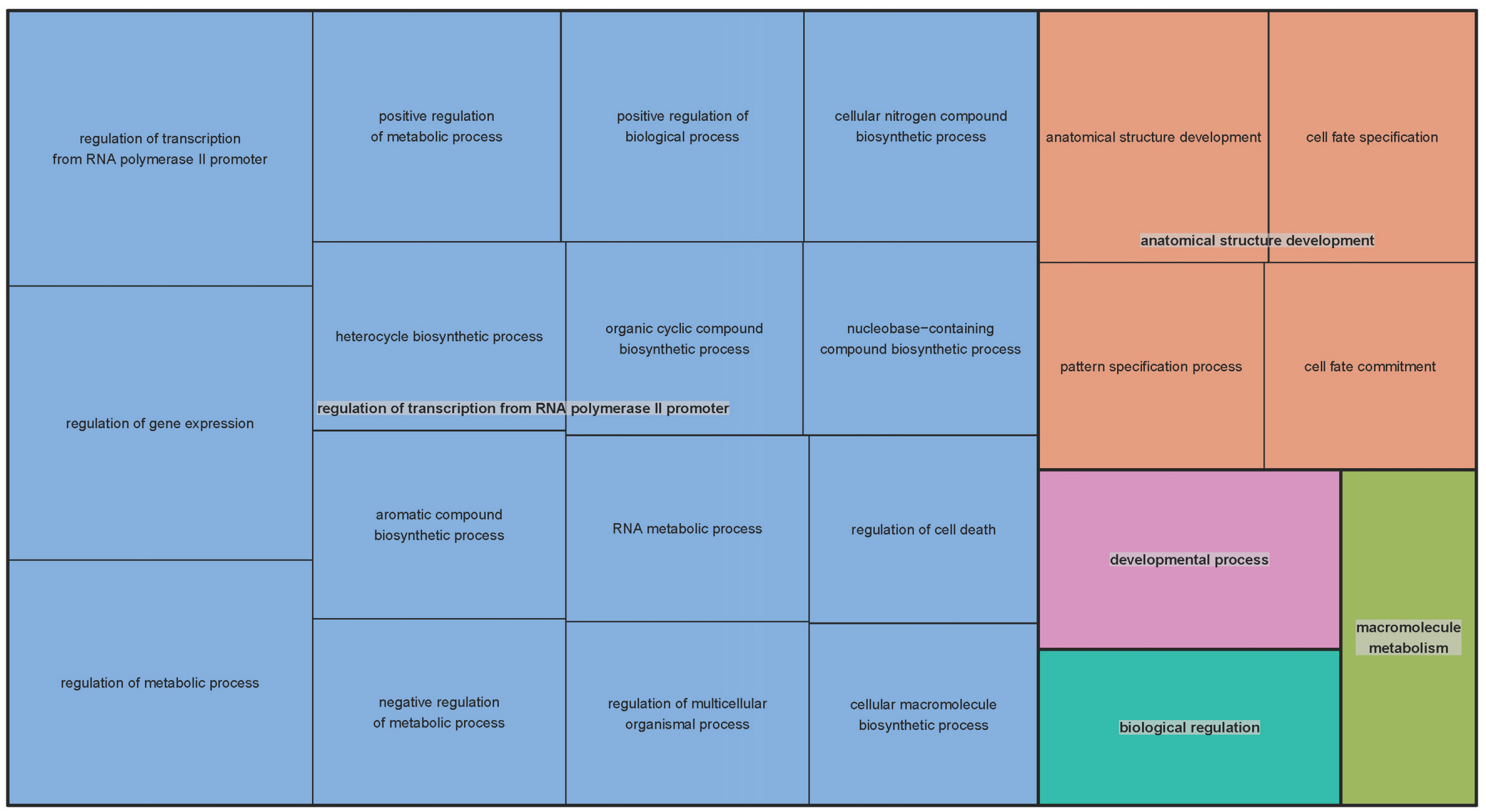

FIGURE 2 | Mouse extended association analysis network and Gene Ontology (GO) analysis of network genes. (A) Association analysis of the 13 mouse VZ network genes. 50 associated genes are shown in the extended network. Black circles indicate the genes that were searched through GeneMANIA and the gray circles are associated genes. The edges are indicated by associations found through co-expression (purple lines), co-localization (blue lines), physical interaction (pink lines), predicted (orange lines) and shared protein domains (tan lines). Table S1 contains complete network details and methods. (B) "TreeMap" view of the GO analysis of 63 genes from the extended mouse network. Rectangles are cluster representatives, which are joined into superclusters of loosely related GO terms and are represented by different colors. The size of the rectangles is a reflection of the adjusted $p$-value of the enrichment of each $\mathrm{GO}$ term relative to the mouse reference list. GO analysis was performed by GOrilla (Eden et al., 2007, 2009) and the "TreeMap" was generated in REViGO (Supek et al., 2011). All GO terms, associated $p$-values and genes for each term can be found in Table S2. 


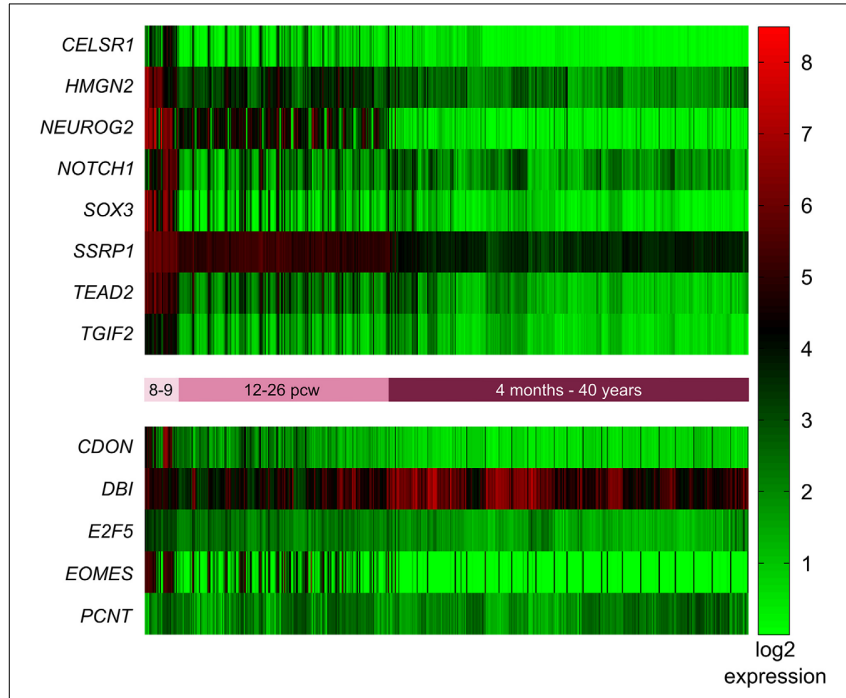

FIGURE 3 | Expression of mouse VZ network genes in different areas of the human developing brain (15-20 post-conception weeks). Genes with significantly higher expression in the ventricular and subventricular zone (VZ and SVZ respectively) compared to all other brain regions were identified using the prenatal laser microdissection (LMD) microarray dataset from the BrainSpan atlas of the developing human brain. Resulting gene lists were then compared to those genes identified in mice. We found that ten of the 13 mouse genes were significantly more highly expressed in the human VZ and SVZ, namely CDON, CELSR1, E2F5, EOMES, HMGN2, NEUROG2, NOTCH1, SOX3, TEAD2, and TGIF2. The other three genes-DBI, PCNT, and SSRP1-were not enriched in the human $V Z$ or $S V Z$. $M Z$, marginal zone; $C P$, cortical plate; SP, subplate zone; IZ, intermediate zone; SZ, subventricular zone; VZ, ventricular zone; AMY, amygdaloid complex; THM, thalamus; Tg, tegmentum; NP, neural plate.

are in the complete list of 407 human genes involved in VZ and SVZ development. This highly substantiates the use of GeneMANIA as a tool for predicting relationships between genes. The four genes that are unique to the extended human network (MCM6, KIF18B, FEN1 and CCNF) are known to be involved in DNA replication, mitosis and cell cycle specific proteasome degradation.

\section{PATHWAY AND GO ANALYSIS}

To gain insight into the function of the 407 genes that are enriched in the human SVZ and VZ, we examined GO and pathway enrichment of these genes. In addition to GOrilla and REViGO (used for the mouse GO analysis) we also used WebGestalt (http://bioinfo. vanderbilt.edu/webgestalt/), which has several enrichment analysis tools (Eden et al., 2007, 2009; Supek et al., 2011; Wang et al., 2013). We found a significant enrichment in genes involved in the cell cycle, DNA replication and specific stages of mitotis. The top 10 pathways that are enriched in this gene list are represented in Table 1. Among the GO enriched terms for biological processes are cell cycle process, mitotic cell process, DNA replication and chromatin assembly and disassembly (Figure 5B and Table S5). The pathway and GO analysis for the human network is consistent with functions expected for genes involved in early cortical development.

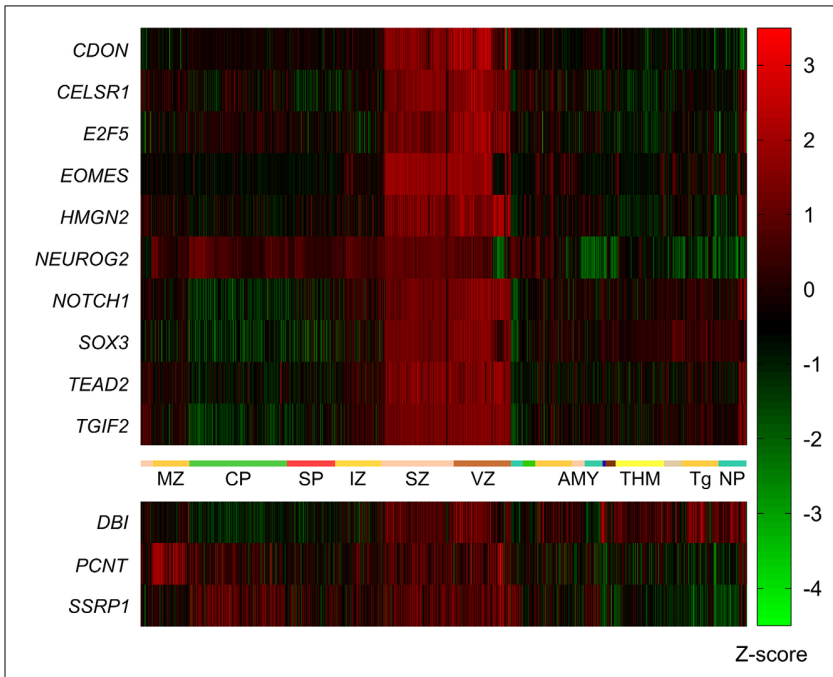

FIGURE 4 | Expression of mouse network genes in the human brain throughout development. Genes with significantly higher expression at post-conception weeks (pcw) 8-9 compared to later time points were analyzed using the developmental transcriptome dataset from the BrainSpan atlas of the developing human brain. Eight of the 13 mouse genes were significantly more highly expressed at 8-9 pcw: CELSR1, HMGN2, NEUROG2, NOTCH1, SOX3, SSRP1, TEAD2, and TGIF2. CDON, $D B I, E 2 F 5, E O M E S$, and $P C N T$ did not show increased expression during 8-9 pcw compared to later stages.

\section{DISCUSSION}

\section{GENERATION OF GENE NETWORKS THAT ARE INVOLVED IN CNS DEVELOPMENTAL PROCESSES}

Development of the CNS is a set of processes that increase the complexity of the brain. Genes are spatially and temporally coordinated to control normal CNS development, while perturbed modulation results in neurological diseases. To understand the regulatory network during these processes, spatio-temporal information of gene expression in the brain is required. In recent decades, spatial mapping of ISH has been a useful tool to demonstrate regional gene expression patterns in different model organisms (De Boer et al., 2009). The Allen Institute for Brain Science provides high resolution ISH datasets containing spatial information at multiple developmental stages of the mouse brain. These datasets allowed us to generate a network composed of correlated genes that are highly expressed in the VZ at early stages of mouse neocortical development (Figure 1). It should be pointed out that only $10 \%$ of the mouse genes exist in the Developing Mouse Brain Atlas. Therefore, we clearly significantly underestimate the number of genes involved in mouse VZ development. Even with this underestimation, novel gene regulators were identified based on the derived gene network. As a second example, we generated a non- $\mathrm{VZ}$ network of genes expressed in regions adjacent to the VZ at the same stage (Figure S1). Note that all of the gene expression patterns shown by the Allen Developing Mouse Brain Atlas are specific to the C57BL/6J mouse strain and, thus, do not address variation along the genetic axis (Peirce and Williams, 2006).

We further performed a cross-species data search with the BrainSpan Atlas of the Developing Human Brain (Miller 
A

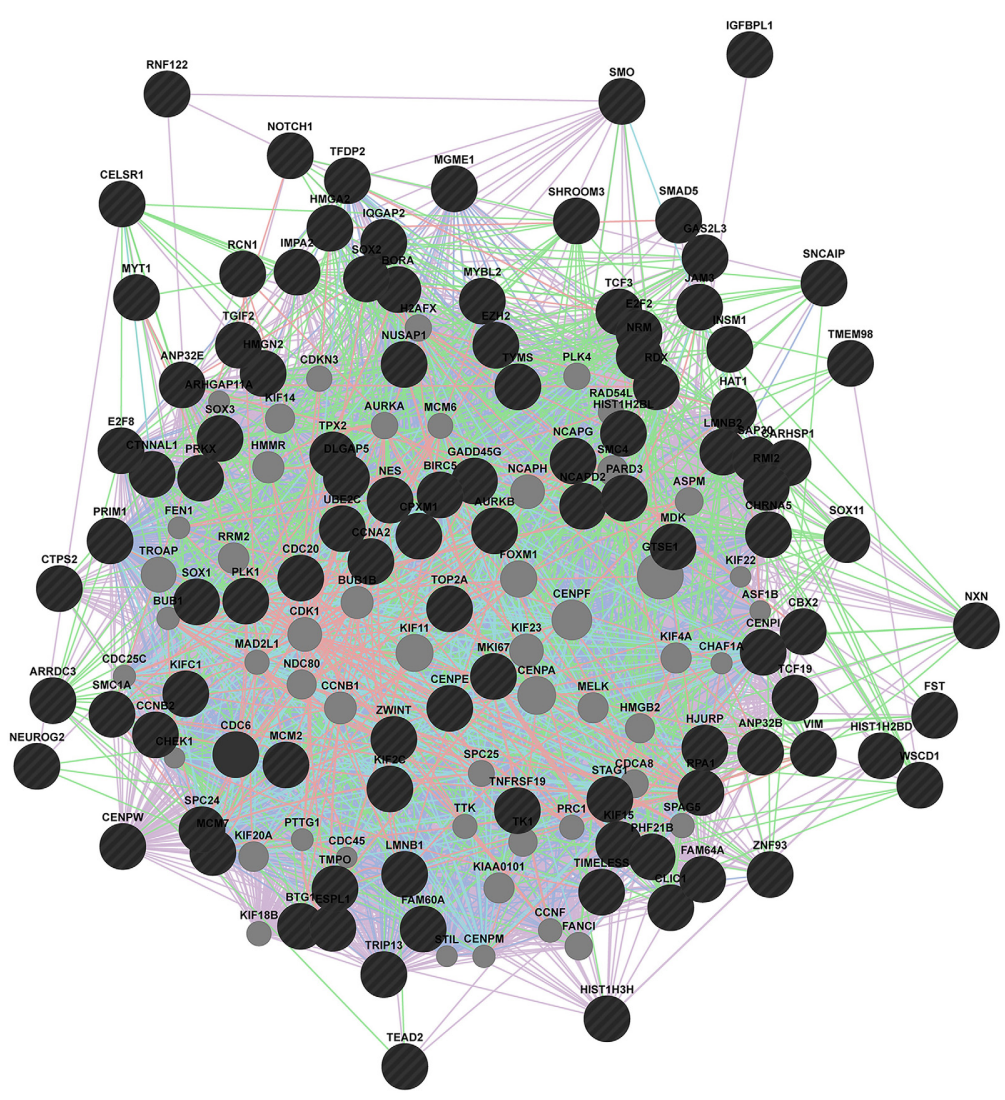

B

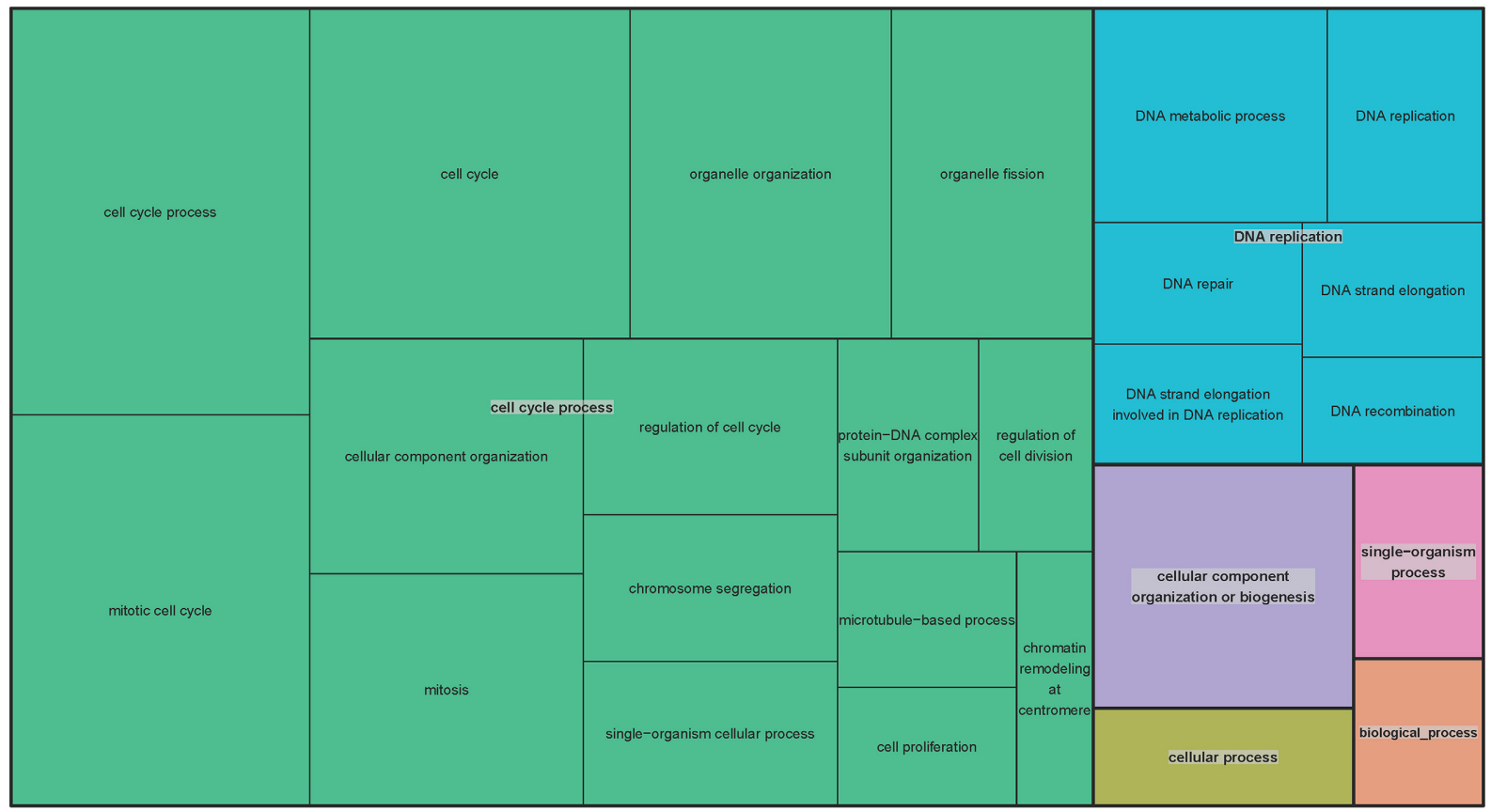

FIGURE 5 | Human extended association analysis network and Gene Ontology (GO) analysis of human network genes. (A) The top 100 genes from the human network and the 7 genes that overlap between the human and mouse network were used to search for associations between those genes and to find additional genes that associate with them. 50 associated genes are shown in the network. Black circles indicate the genes that were searched through GeneMANIA and the gray circles are associated genes. The edges are indicated by associations found through co-expression (purple lines), co-localization (blue lines), physical interaction (pink lines) and shared

(Continued) 


\section{FIGURE 5 | Continued}

protein domains (tan lines). Predicted associations were not included in the human network due to the large amount of genes. Table S4 contains complete network details and methods. (B) "TreeMap" view of the GO analysis of 407 genes that are expressed at early stages of human VZ and SVZ development. Rectangles are cluster representatives, which are joined into superclusters of loosely related GO terms and are represented by different colors. The size of the rectangles are a reflection of the adjusted $p$-value of the enrichment of each GO term relative to the human reference list. GO analysis was performed by GOrilla (Eden et al., 2007, 2009) and the "TreeMap" was generated in REViGO (Supek et al., 2011). All GO terms, associated $p$-values and genes for each term can be found in Table S5.

Table 1 | Pathway enrichment analysis of genes highly enriched in the ventricular and subventricular zone during early human development using WebGestalt.

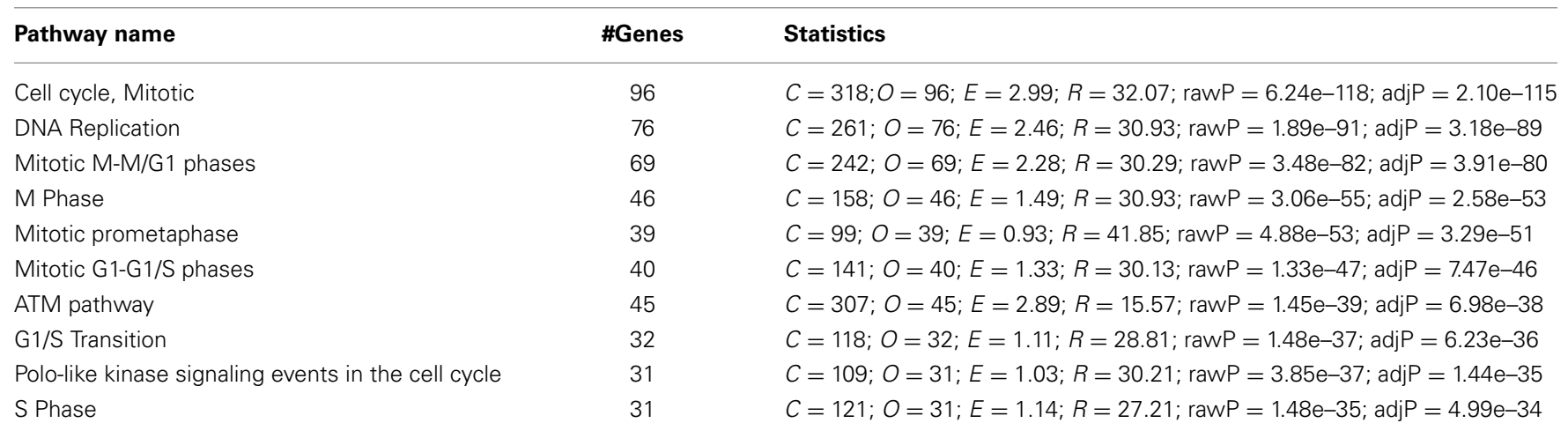

A list of 407 genes was provided to WebGestalt and the predefined human reference genome was used as the reference set. The top 10 most significantly enriched pathways are shown with an adjusted p-value of $4.99 \times 10^{-34}$ or lower. $C$, number of reference genes in category; $O$, number of genes in the category from the gene set; $E$, expected number in category; $R$, ratio of enrichment; rawP, p-value from hypergeometric test; adjp, $p$-value adjusted by the multiple test adjustment.

et al., 2014), specifically of the VZ and SVZ of the neocortex (Figures 3, 4). The standardized bioinformatics pipeline employed by the Allen Brain Atlas enabled the usage of data from different sources and the combination of data across different species (Sunkin et al., 2013). Translating developmental time between mice and human, we chose comparable time points to analyze the human data that were used earlier to compile the mouse neocortical data (Clancy et al., 2001). The increased sensitivity of the methodology in the Human Developing Atlas, which uses next generation sequencing and microarray technologies, allows identifications of more regulators of neocortical development. This integrated cross-species approach is a powerful tool to fully exploit stored gene expression data, and unravel the gene regulatory networks during brain development with both spatial and temporal information.

\section{IDENTIFICATION OF NOVEL GENE REGULATORS AND FUNCTIONS DURING NEOCORTICAL DEVELOPMENT}

The growth of the cerebral cortex largely depends on the proliferation of neural progenitors, and the subsequent generation of differentiated neural cells. Consistent with this, GO analysis of the mouse VZ network and extended network suggests involvement in cell fate specification and commitment, regulation of transcription and developmental processes. For example, Notch1 regulates cell cycle progression, which maintains the stem population in both the subgranular zone of the dentate gyrus and the SVZ (Aguirre et al., 2010; Ables et al., 2011). The acquisition of radial glia and astrocytes with stem cell properties is also promoted by Notch1 (Gaiano et al., 2000), while neuronal and oligodendrocyte differentiation are inhibited (Wang et al., 1998; Yoon and Gaiano, 2005). Later in development, altered expression levels of Notch1 affect the morphology of neurons, and perturb the neuronal migration in the cerebral cortex (Hashimoto-Torii et al., 2008). Sox3, another candidate gene, has a function during brain development similar to that of Notch1. Notably, several studies performed in non-mammalian species indicate that Sox3 negatively regulates neurogenesis to maintain the stemness of precursors (Bylund et al., 2003; Archer et al., 2011).

Some of our candidate genes are known to be involved in neurogenesis and neuronal migration, which are essential processes in the development of the cerebral cortex. Neurog2 is a proneural transcription factor initiating neuronal differentiation (Wilkinson et al., 2013). When overexpressed Neurog2 promotes neurogenesis more rapidly in dorsal vs. ventral telencephalic progenitor cells specifically at E12.5 in mice (Li et al., 2012a), suggesting the modulation of Neurog2 is spatially and temporally regulated. Interestingly, Neurog2 is one of the four genes (Cdon, Celsr1, Eomes, and Neurog2) specifically reduced in the mouse GE compared with the VZ of the neocortex (Figures 1B,C,F,H). The GE emerges early in telencephalic development. Cells from the GE migrate tangentially and supply inhibitory neurons (i.e., GABAergic neurons) to the neocortex. Indeed, Neurog2 represses the generation of the GABAergic neuronal phenotype whereas it promotes the generation of glutamatergic neurons when facing the binary cell fate decision (Schuurmans et al., 2004). Another gene that we found to be downregulated in the mouse GE is Eomes, which we also found to be specifically expressed in the human VZ and SVZ early in development (however note that expression of EOMES 8-9 pcw compared to all later stages was only nominally significant and did not survive correction for multiple testing) (Figures 3, 4). Similar to Neurog2, Eomes promotes neuronal-fate commitment (Hodge et al., 2012). Studies 
demonstrate that Eomes might be required to control the equilibrium between glutamatergic and GABAergic neurons that migrate either to the olfactory bulb (Mizuguchi et al., 2012) or cerebral cortex (Sessa et al., 2010). Celsr1 is implicated in both tangential and radial migration as well, in facial branchiomotor neurons (Boutin et al., 2012). Hence, our results imply that Cdon and Celsr1 are likely to be involved in regulating the size of the excitatory-inhibitory neuronal pool.

Only limited data is available for the other candidate genes (Tead2, Tgif2, Cdon, Dbi, Pcnt, E2f5, Hmgn2, and Ssrp1) during early neural development. Tead2 is a transcription factor that is essential for neural tube closure (Kaneko et al., 2007), probably via regulating cell proliferation and apoptosis (Sawada et al., 2008). The contribution from Tgif2 in holoprosencephaly (failure of the embryonic forebrain to completely divide into two hemispheres) is still controversial (Shen and Walsh, 2005; Taniguchi et al., 2012). Notably, other genes not in our combined human and mouse candidate list, including Cdon, Dbi and Pcnt, also exhibit potential effects during neural development (Zhang et al., 2006; Oh et al., 2009; Buchman et al., 2010; Endoh-Yamagami et al., 2010; Alfonso et al., 2012). Loss of E2f5 does not perturb cell proliferation in the $\mathrm{VZ}$ during early development in mice (Lindeman et al., 1998), which might be due to the compensating effect of other E2F transcription factors (e.g., E2f1) (Yoshikawa, 2000; Cooper-Kuhn et al., 2002). Hmgn2 and Ssrp1 are both chromatin binding proteins that are novel potential regulators of neocortical development implicated by our examination of a publically available dataset. This is also supported by their involvement in DNA replication and cell cycle progression (Cherukuri et al., 2008; Formosa, 2012).

\section{VALIDATION OF GENEMANIA GENERATED NETWORK BY LITERATURE MINING}

Potential cross-talk among our candidate genes was verified by literature mining. We made our space-time VZ network (Figure 1) into a more complex and hypothetical functional gene network (Figure 6). In line with GeneMANIA analysis, Neurog2, Eomes and Notch1 closely interact with each other. In fact, Eomes and Neurog2 have similar expression patterns in the cortical progenitors of the VZ, which is initiated by direct binding of NEUROG2 to the promoter of Eomes (Ochiai et al., 2009). In addition, these two genes interact via co-regulators of transcription. Pax6 negatively regulates the expression of Eomes (Imamura and Greer, 2013), while upregulating Neurog2 during early neural development (Scardigli, 2003). As mentioned earlier, Notch1 exhibits opposite effects in neurogenesis compared with Neurog2 and Eomes. Consistently, the Neurog2-Eomes cascade is suppressed by Notch signaling through Hes 1 with a positive feedback from Neurog2 via the Notch ligand Dll1 (Kageyama et al., 2008). The correlation between these genes is also facilitated by the canonical Wnt signaling pathway (Shimizu et al., 2008; Li et al., 2012a). Coordinated interplay among the genes in the network controls the switch between neural stem cells and fate-committed neurons, balancing the pool of each neural population during development.

In contrast, Hmgn2 displays little interaction with other genes, also shown by the GeneMANIA network (Figure 2A).

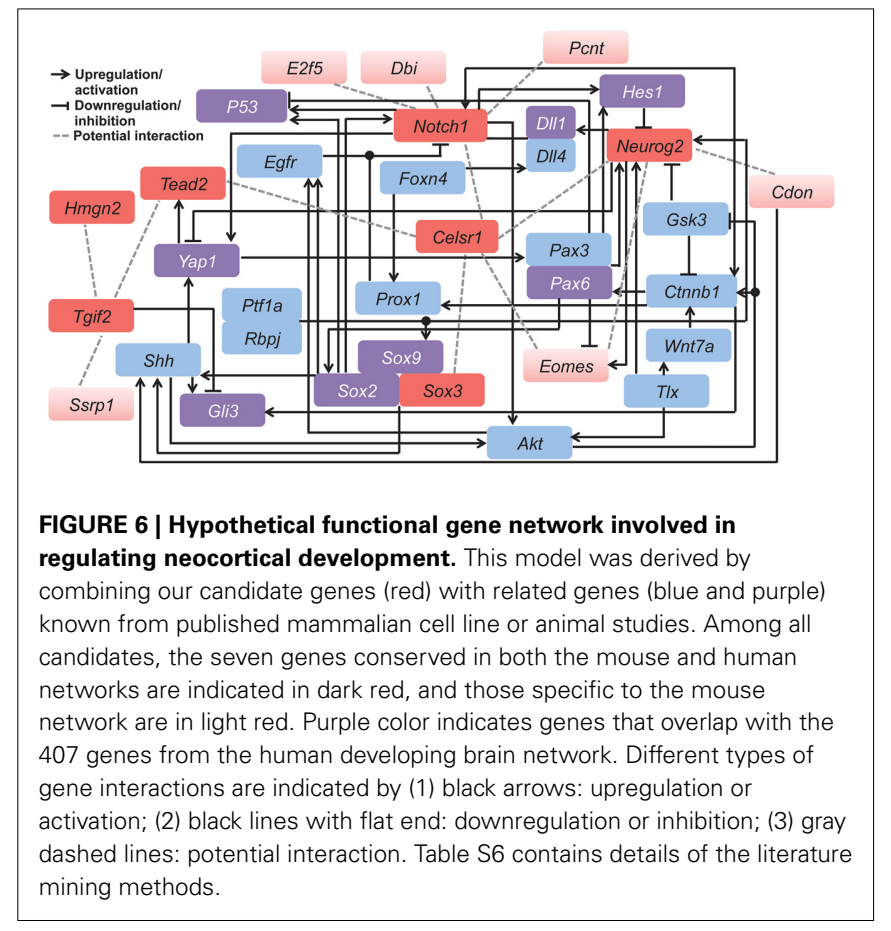

This further supports the hypothesis that Hmgn2 is a novel regulator in this network. In addition, some connections indicated by our hypothetical functional gene network are novel. For instance, Celsr1 shows little interaction with other candidates in previous research. However, it is a central component in our mouse Neocortex VZ Network, and its involvement is conserved in the human network. The interplay between Celsrl and other genes are also predicted in both the mouse and human extended networks (Figures 2A, 5A). Thus, Celsr1 could be a promising target for future neural developmental research. In addition, four of the 28 genes from the hypothetical network overlap with the GeneMANIA extended mouse network (i.e., Shh, Smad2, Sox2, and Yap1) (Tables S1,S6), and eight genes overlap with the 407 genes from the human developing brain network (i.e., DLL1, GLI3, HES1, TP53, PAX6, SOX2, SOX9, and YAP1) (Tables S4,S6). The consistency between the literature mining and GeneMANIA results validates that GeneMANIA can be a powerful tool in unmasking potential genetic interactions.

Taken together, our proposed gene networks provide a comprehensive view to discover genes involved in complex developmental processes, to reveal the relationships among them, and to help understand genetic modulation at a systematic level. Specifically, through the use of databases that incorporate multiple datasets from both human and mouse studies (Allen Brain Atlas and GeneMANIA) and the analysis of the extensive literature on the developing neocortex, we have uncovered at least two genes (Hmgn2 and Ssrp1) from our mouse network not previously known to be involved in neocortical development. In addition, the data from the human indicates 100's of genes potentially involved in ventricular zone development that could be the basis for future investigations. 


\section{ACKNOWLEDGMENTS}

We thank the International Neuroinformatics Coordinating Facility (www.incf.org), and the University of Tennessee Center for Integrative and Translational Genomics for their support of the 2013 Neuroinformatics Jamboree. This study has been supported in part by the Alexander von Humboldt foundation (Florian Freudenberg), the FSU Department of Biomedical Sciences (Richard S. Nowakowski, Cynthia M. Vied), FCT, Fundação para a Ciência e Tecnologia PTDC/NEUNMC/0315/2012 (Alexandre A. S. F. Raposo).

\section{SUPPLEMENTARY MATERIAL}

The Supplementary Material for this article can be found online at: http://www.frontiersin.org/journal/10.3389/fnins. 2014.00257/abstract

\section{REFERENCES}

Ables, J. L., Breunig, J. J., Eisch, A. J., and Rakic, P. (2011). Not(ch) just development: notch signalling in the adult brain. Nat. Rev. Neurosci. 12, 269-283. doi: $10.1038 / \mathrm{nrn} 3024$

Aguirre, A., Rubio, M. E., and Gallo, V. (2010). Notch and EGFR pathway interaction regulates neural stem cell number and self-renewal. Nature 467, 323-327. doi: 10.1038/nature09347

Alfonso, J., Le Magueresse, C., Zuccotti, A., Khodosevich, K., and Monyer, H. (2012). Diazepam binding inhibitor promotes progenitor proliferation in the postnatal SVZ by reducing GABA signaling. Cell Stem Cell 10, 76-87. doi: 10.1016/j.stem.2011.11.011

Allen, B. L., Tenzen, T., and McMahon, A. P. (2007). The Hedgehog-binding proteins Gas1 and Cdo cooperate to positively regulate Shh signaling during mouse development. Genes Dev. 21, 1244-1257. doi: 10.1101/gad.15 43607

Archer, T. C., Jin, J., and Casey, E. S. (2011). Interaction of Sox1, Sox2, Sox3 and Oct4 during primary neurogenesis. Dev. Biol. 350, 429-440. doi: 10.1016/j.ydbio.2010.12.013

Ayala, R., Shu, T., and Tsai, L.-H. (2007). Trekking across the brain: the journey of neuronal migration. Cell 128, 29-43. doi: 10.1016/j.cell.2006.12.021

Boutin, C., Goffinet, A. M., and Tissir, F. (2012). Celsr1-3 cadherins in PCP and brain development. Curr. Top. Dev. Biol. 101, 161-183. doi: 10.1016/B978-0-12394592-1.00010-7

Buchman, J. J., Tseng, H.-C., Zhou, Y., Frank, C. L., Xie, Z., and Tsai, L.-H. (2010). Cdk5rap2 interacts with pericentrin to maintain the neural progenitor pool in the developing neocortex. Neuron 66, 386-402. doi: 10.1016/j.neuron.2010.03.036

Bylund, M., Andersson, E., Novitch, B. G., and Muhr, J. (2003). Vertebrate neurogenesis is counteracted by Sox1-3 activity. Nat. Neurosci. 6, 1162-1168. doi: $10.1038 / \mathrm{nn} 1131$

Caviness, V. S., Nowakowski, R. S., and Bhide, P. G. (2009). Neocortical neurogenesis: morphogenetic gradients and beyond. Trends Neurosci. 32, 443-450. doi: 10.1016/j.tins.2009.05.003

Cerami, E. G., Gross, B. E., Demir, E., Rodchenkov, I., Babur, O., Anwar, N., et al. (2011). Pathway Commons, a web resource for biological pathway data. Nucleic Acids Res. 39, D685-D690. doi: 10.1093/nar/gkq1039

Chavali, P. L., Saini, R. K. R., Matsumoto, Y., Ågren, H., and Funa, K. (2011). Nuclear orphan receptor TLX induces Oct-3/4 for the survival and maintenance of adult hippocampal progenitors upon hypoxia. J. Biol. Chem. 286, 9393-9404. doi: 10.1074/jbc.M110.167445

Cherukuri, S., Hock, R., Ueda, T., Catez, F., Rochman, M., and Bustin, M. (2008). Cell cycle-dependent binding of HMGN proteins to chromatin. Mol. Biol. Cell 19, 1816-1824. doi: 10.1091/mbc.E07-10-1018

Clancy, B., Darlington, R. B., and Finlay, B. L. (2001). Translating developmental time across mammalian species. Neuroscience 105, 7-17. doi: 10.1016/S03064522(01)00171-3

Cooper-Kuhn, C., Vroemen, M., Brown, J., Ye, H., Thompson, M., Winkler, J., et al. (2002). Impaired adult neurogenesis in mice lacking the transcription factor E2F1. Mol. Cell. Neurosci. 21, 312-323. doi: 10.1006/mcne.2002.1176
De Boer, B. A., Ruijter, J. M., Voorbraak, F. P. J. M., and Moorman, A. F. M. (2009). More than a decade of developmental gene expression atlases: where are we now? Nucleic Acids Res. 37, 7349-7359. doi: 10.1093/nar/gkp819

De la Pompa, J. L., Wakeham, A., Correia, K. M., Samper, E., Brown, S., Aguilera, R. J., et al. (1997). Conservation of the Notch signalling pathway in mammalian neurogenesis. Development 124, 1139-1148.

Eden, E., Lipson, D., Yogev, S., and Yakhini, Z. (2007). Discovering motifs in ranked lists of DNA sequences. PLoS Comput. Biol. 3:e39. doi: 10.1371/journal.pcbi.0030039

Eden, E., Navon, R., Steinfeld, I., Lipson, D., and Yakhini, Z. (2009). GOrilla: a tool for discovery and visualization of enriched GO terms in ranked gene lists. $B M C$ Bioinformatics 10:48. doi: 10.1186/1471-2105-10-48.

Endoh-Yamagami, S., Karkar, K. M., May, S. R., Cobos, I., Thwin, M. T., Long, J. E., et al. (2010). A mutation in the pericentrin gene causes abnormal interneuron migration to the olfactory bulb in mice. Dev. Biol. 340, 41-53. doi: 10.1016/j.ydbio.2010.01.017

Favaro, R., Valotta, M., Ferri, A. L. M., Latorre, E., Mariani, J., Giachino, C., et al. (2009). Hippocampal development and neural stem cell maintenance require Sox2-dependent regulation of Shh. Nat. Neurosci. 12, 1248-1256. doi: $10.1038 / \mathrm{nn} .2397$

Fernandez,-L. A., Northcott, P. A., Dalton, J., Fraga, C., Ellison, D., Angers, S., et al. (2009). YAP1 is amplified and up-regulated in hedgehog-associated medulloblastomas and mediates Sonic hedgehog-driven neural precursor proliferation. Genes Dev. 23, 2729-2741. doi: 10.1101/gad.1824509

Formosa, T. (2012). The role of FACT in making and breaking nucleosomes. Biochim. Biophys. Acta 1819, 247-255. doi: 10.1016/j.bbagrm.2011.07.009

Gaiano, N., Nye, J. S., and Fishell, G. (2000). Radial glial identity is promoted by Notch1 signaling in the murine forebrain. Neuron 26, 395-404. doi: 10.1016/S0896-6273(00)81172-1

Gee, S. T., Milgram, S. L., Kramer, K. L., Conlon, F. L., and Moody, S. A. (2011). Yes-associated protein 65 (YAP) expands neural progenitors and regulates Pax3 expression in the neural plate border zone. PLoS ONE 6:e20309. doi: 10.1371/journal.pone.0020309

Götz, M., and Huttner, W. B. (2005). The cell biology of neurogenesis. Nat. Rev. Mol. Cell Biol. 6, 777-788. doi: 10.1038/nrm1739

Hartfuss, E., Galli, R., Heins, N., and Götz, M. (2001). Characterization of CNS precursor subtypes and radial glia. Dev. Biol. 229, 15-30. doi: 10.1006/dbio.2000.9962

Hashimoto-Torii, K., Torii, M., Sarkisian, M. R., Bartley, C. M., Shen, J., Radtke, F., et al. (2008). Interaction between Reelin and Notch signaling regulates neuronal migration in the cerebral cortex. Neuron 60, 273-284. doi: 10.1016/j.neuron.2008.09.026

Haubensak, W., Attardo, A., Denk, W., and Huttner, W. B. (2004). Neurons arise in the basal neuroepithelium of the early mammalian telencephalon: a major site of neurogenesis. Proc. Natl. Acad. Sci. U.S.A. 101, 3196-3201. doi: 10.1073/pnas.0308600100

Henke, R. M., Savage, T. K., Meredith, D. M., Glasgow, S. M., Hori, K., Dumas, J., et al. (2009). Neurog2 is a direct downstream target of the Ptfla-Rbpj transcription complex in dorsal spinal cord. Development 136, 2945-2954. doi: $10.1242 /$ dev. 035352

Hodge, R. D., Nelson, B. R., Kahoud, R. J., Yang, R., Mussar, K. E., Reiner, S. L., et al. (2012). Tbr2 is essential for hippocampal lineage progression from neural stem cells to intermediate progenitors and neurons. J. Neurosci. 32, 6275-6287. doi: 10.1523/JNEUROSCI.0532-12.2012

Hu, Q., Zhang, L., Wen, J., Wang, S., Li, M., Feng, R., et al. (2010). The EGF receptor-sox2-EGF receptor feedback loop positively regulates the self-renewal of neural precursor cells. Stem Cells 28, 279-286. doi: 10.1002/stem.246

Huttner, W. B., and Kosodo, Y. (2005). Symmetric versus asymmetric cell division during neurogenesis in the developing vertebrate central nervous system. Curr. Opin. Cell Biol. 17, 648-657. doi: 10.1016/j.ceb.2005.10.005

Imamura, F., and Greer, C. A. (2013). Pax6 regulates Tbr1 and Tbr2 expressions in olfactory bulb mitral cells. Mol. Cell. Neurosci. 54, 58-70. doi: 10.1016/j.mcn.2013.01.002

Johansson, P. A., Cappello, S., and Götz, M. (2010). Stem cells niches during development-lessons from the cerebral cortex. Curr. Opin. Neurobiol. 20, 400-407. doi: 10.1016/j.conb.2010.04.003

Kageyama, R., Ohtsuka, T., Shimojo, H., and Imayoshi, I. (2008). Dynamic Notch signaling in neural progenitor cells and a revised view of lateral inhibition. Nat. Neurosci. 11, 1247-1251. doi: 10.1038/nn.2208 
Kaltezioti, V., Kouroupi, G., Oikonomaki, M., Mantouvalou, E., Stergiopoulos, A., Charonis, A., et al. (2010). Proxl regulates the notchl-mediated inhibition of neurogenesis. PLoS Biol. 8:e1000565. doi: 10.1371/journal.pbio. 1000565

Kaneko, K. J., Kohn, M. J., Liu, C., and DePamphilis, M. L. (2007). Transcription factor TEAD2 is involved in neural tube closure. Genesis 45, 577-587. doi: 10.1002/dvg.20330

Karalay, O., Doberauer, K., Vadodaria, K. C., Knobloch, M., Berti, L., Miquelajauregui, A., et al. (2011). Prospero-related homeobox 1 gene (Prox1) is regulated by canonical Wnt signaling and has a stage-specific role in adult hippocampal neurogenesis. Proc. Natl. Acad. Sci. U. S. A. 108, 5807-5812. doi: 10.1073/pnas.1013456108

Kowalczyk, T., Pontious, A., Englund, C., Daza, R. A. M., Bedogni, F., Hodge, R., et al. (2009). Intermediate neuronal progenitors (basal progenitors) produce pyramidal-projection neurons for all layers of cerebral cortex. Cereb. Cortex 19, 2439-2450. doi: 10.1093/cercor/bhn260

Kriegstein, A. R., and Götz, M. (2003). Radial glia diversity: a matter of cell fate. Glia 43, 37-43. doi: 10.1002/glia.10250

Li, S., Mattar, P., Zinyk, D., Singh, K., Chaturvedi, C.-P., Kovach, C., et al. (2012a). GSK3 temporally regulates neurogenin 2 proneural activity in the neocortex. J. Neurosci. 32, 7791-7805. doi: 10.1523/JNEUROSCI.1309-12.2012

Li, S., Mo, Z., Yang, X., Price, S. M., Shen, M. M., and Xiang, M. (2004). Foxn4 controls the genesis of amacrine and horizontal cells by retinal progenitors. Neuron 43, 795-807. doi: 10.1016/j.neuron.2004.08.041

Li, Y., Hibbs, M. A., Gard, A. L., Shylo, N. A., and Yun, K. (2012b). Genome-wide analysis of N1ICD/RBPJ targets in vivo reveals direct transcriptional regulation of Wnt, SHH, and hippo pathway effectors by Notch1. Stem Cells 30, 741-752. doi: 10.1002/stem.1030

Lindeman, G. J., Dagnino, L., Gaubatz, S., Xu, Y., Bronson, R. T., Warren, H. B., et al. (1998). A specific, nonproliferative role for E2F-5 in choroid plexus function revealed by gene targeting. Genes Dev. 12, 1092-1098. doi: 10.1101/gad.12.8.1092

Marqués-Torrejón, M. Á., Porlan, E., Banito, A., Gómez-Ibarlucea, E., LopezContreras, A. J., Fernández-Capetillo, O., et al. (2013). Cyclin-dependent kinase inhibitor p21 controls adult neural stem cell expansion by regulating Sox2 gene expression. Cell Stem Cell 12, 88-100. doi: 10.1016/j.stem.2012. 12.001

Miller, J. A., Sunkin, S. M., Smith, K. A., Ng, L., Szafer, A., Ebbert, A., et al. (2014). Transcriptional landscape of the prenatal human brain. Nature 508, 199-206. doi: 10.1038/nature13185

Misra, K., Luo, H., Li, S., Matise, M., and Xiang, M. (2014). Asymmetric activation of Dll4-Notch signaling by Foxn 4 and proneural factors activates BMP/TGF $\beta$ signaling to specify V2b interneurons in the spinal cord. Development 141, 187-198. doi: 10.1242/dev.092536

Miyoshi, K., Onishi, K., Asanuma, M., Miyazaki, I., Diaz-Corrales, F. J., and Ogawa, N. (2006). Embryonic expression of pericentrin suggests universal roles in ciliogenesis. Dev. Genes Evol. 216, 537-542. doi: 10.1007/s00427-0060065-8.

Mizuguchi, R., Naritsuka, H., Mori, K., Mao, C.-A., Klein, W. H., and Yoshihara, Y. (2012). Tbr2 deficiency in mitral and tufted cells disrupts excitatory-inhibitory balance of neural circuitry in the mouse olfactory bulb. J. Neurosci. 32, 8831-8844. doi: 10.1523/JNEUROSCI.5746-11.2012

Morin, X., and Bellaïche, Y. (2011). Mitotic spindle orientation in asymmetric and symmetric cell divisions during animal development. Dev. Cell 21, 102-119. doi: 10.1016/j.devcel.2011.06.012

Mostafavi, S., Ray, D., Warde-Farley, D., Grouios, C., and Morris, Q. (2008). GeneMANIA: a real-time multiple association network integration algorithm for predicting gene function. Genome Biol. 9 (Suppl. 1), S4. doi: 10.1186/gb2008-9-s1-s4

Nakazaki, H., Reddy, A. C., Mania-Farnell, B. L., Shen, Y.-W., Ichi, S., McCabe, C., et al. (2008). Key basic helix-loop-helix transcription factor genes Hes 1 and Ngn2 are regulated by Pax3 during mouse embryonic development. Dev. Biol. 316, 510-523. doi: 10.1016/j.ydbio.2008.01.008

Neumüller, R. A., Richter, C., Fischer, A., Novatchkova, M., Neumüller, K. G., and Knoblich, J. A. (2011). Genome-wide analysis of self-renewal in Drosophila neural stem cells by transgenic RNAi. Cell Stem Cell 8, 580-593. doi: 10.1016/j.stem.2011.02.022

Ochiai, W., Nakatani, S., Takahara, T., Kainuma, M., Masaoka, M., Minobe, S., et al. (2009). Periventricular notch activation and asymmetric Ngn2 and Tbr2 expression in pair-generated neocortical daughter cells. Mol. Cell. Neurosci. 40, 225-233. doi: 10.1016/j.mcn.2008.10.007

Oh, J.-E., Bae, G.-U., Yang, Y.-J., Yi, M.-J., Lee, H.-J., Kim, B.-G., et al. (2009). Cdo promotes neuronal differentiation via activation of the p38 mitogen-activated protein kinase pathway. FASEB J. 23, 2088-2099. doi: 10.1096/fj.08-119255

Pani, L., Horal, M., and Loeken, M. R. (2002). Rescue of neural tube defects in Pax-3-deficient embryos by p53 loss of function: implications for Pax3- dependent development and tumorigenesis. Genes Dev. 16, 676-680. doi: $10.1101 /$ gad. 969302

Peirce, J. L., and Williams, R. W. (2006). "Expression and brain structure: black boxes between genes and behaviors," in Neurobehavioral Genetics: Methods and Applications, 2nd Edn, eds B. C. Jones and P. Mormede (Boca Raton, FL: CRC Press), 411-434.

Petrova, R., Garcia, A. D. R., and Joyner, A. L. (2013). Titration of GLI3 repressor activity by sonic hedgehog signaling is critical for maintaining multiple adult neural stem cell and astrocyte functions. J. Neurosci. 33, 17490-17505. doi: 10.1523/JNEUROSCI.2042-13.2013

Qu, Q., Sun, G., Li, W., Yang, S., Ye, P., Zhao, C., et al. (2010). Orphan nuclear receptor TLX activates Wnt/beta-catenin signalling to stimulate neural stem cell proliferation and self-renewal. Nat. Cell Biol. 12, 31-39. doi: 10.1038/ ncb2001

Qu, Q., Sun, G., Murai, K., Ye, P., Li, W., Asuelime, G., et al. (2013). Wnt7a regulates multiple steps of neurogenesis. Mol. Cell. Biol. 33, 2551-2559. doi: 10.1128/MCB.00325-13

Roybon, L., Mastracci, T. L., Ribeiro, D., Sussel, L., Brundin, P., and Li, J.-Y. (2010). GABAergic differentiation induced by Mash1 is compromised by the bHLH proteins Neurogenin2, NeuroD1, and NeuroD2. Cereb. Cortex 20, 1234-1244. doi: 10.1093/cercor/bhp187

Sawada, A., Kiyonari, H., Ukita, K., Nishioka, N., Imuta, Y., and Sasaki, H. (2008). Redundant roles of Tead 1 and Tead 2 in notochord development and the regulation of cell proliferation and survival. Mol. Cell. Biol. 28, 3177-3189. doi: 10.1128/MCB.01759-07

Scardigli, R. (2003). Direct and concentration-dependent regulation of the proneural gene Neurogenin2 by Pax6. Development 130, 3269-3281. doi: 10.1242/dev.00539

Schuurmans, C., Armant, O., Nieto, M., Stenman, J. M., Britz, O., Klenin, N., et al. (2004). Sequential phases of cortical specification involve Neurogenindependent and -independent pathways. EMBO J. 23, 2892-2902. doi: 10.1038/sj.emboj.7600278

Sessa, A., Mao, C.-A., Colasante, G., Nini, A., Klein, W. H., and Broccoli, V. (2010). Tbr2-positive intermediate (basal) neuronal progenitors safeguard cerebral cortex expansion by controlling amplification of pallial glutamatergic neurons and attraction of subpallial GABAergic interneurons. Genes Dev. 24, 1816-1826. doi: $10.1101 /$ gad. 575410

Shen, J., and Walsh, C. A. (2005). Targeted disruption of Tgif, the mouse ortholog of a human holoprosencephaly gene, does not result in holoprosencephaly in mice. Mol. Cell. Biol. 25, 3639-3647. doi: 10.1128/MCB.25.9.3639-3647.2005

Shimizu, T., Kagawa, T., Inoue, T., Nonaka, A., Takada, S., Aburatani, H., et al. (2008). Stabilized beta-catenin functions through TCF/LEF proteins and the Notch/RBP-Jkappa complex to promote proliferation and suppress differentiation of neural precursor cells. Mol. Cell. Biol. 28, 7427-7441. doi 10.1128/MCB.01962-07

Shimojo, H., Ohtsuka, T., and Kageyama, R. (2008). Oscillations in notch signaling regulate maintenance of neural progenitors. Neuron 58, 52-64. doi: 10.1016/j.neuron.2008.02.014

Sinor-Anderson, A., and Lillien, L. (2011). Akt1 interacts with epidermal growth factor receptors and hedgehog signaling to increase stem/transit amplifying cells in the embryonic mouse cortex. Dev. Neurobiol. 71, 759-771. doi: 10.1002/dneu.20878

Sun, T., and Hevner, R. F. (2014). Growth and folding of the mammalian cerebral cortex: from molecules to malformations. Nat. Rev. Neurosci. 15, 217-232. doi: 10.1038/nrn3707

Sunkin, S. M., Ng, L., Lau, C., Dolbeare, T., Gilbert, T. L., Thompson, C. L., et al. (2013). Allen brain atlas: an integrated spatio-temporal portal for exploring the central nervous system. Nucleic Acids Res. 41, D996-D1008. doi: 10.1093/nar/gks1042

Supek, F., Bošnjak, M., Škunca, N., and Šmuc, T. (2011). REVIGO summarizes and visualizes long lists of gene ontology terms. PLOS ONE 6:e21800. doi: 10.1371/journal.pone.0021800 
Takahashi, T., Goto, T., Miyama, S., Nowakowski, R. S., and Caviness, V. S. (1999). Sequence of neuron origin and neocortical laminar fate: relation to cell cycle of origin in the developing murine cerebral wall. J. Neurosci. 19, 10357-10371.

Takahashi, T., Nowakowski, R. S., and Caviness, V. S. (1994). Mode of cell proliferation in the developing mouse neocortex. Proc. Natl. Acad. Sci. U.S.A. 91, 375-379. doi: 10.1073/pnas.91.1.375

Takahashi, T., Nowakowski, R. S., and Caviness, V. S. (1995). Early ontogeny of the secondary embryonic murine cerebral wall proliferative population of the. J. Neurosci. 15, 6058-6068.

Taniguchi, K., Anderson, A. E., Sutherland, A. E., and Wotton, D. (2012). Loss of Tgif function causes holoprosencephaly by disrupting the $\mathrm{SHH}$ signaling pathway. PLoS Genet. 8:e1002524. doi: 10.1371/journal.pgen. 1002524

Taranova, O. V., Magness, S. T., Fagan, B. M., Wu, Y., Surzenko, N., Hutton, S. R., et al. (2006). SOX2 is a dose-dependent regulator of retinal neural progenitor competence. Genes Dev. 20, 1187-1202. doi: 10.1101/gad. 1407906

Taylor, M. K., Yeager, K., and Morrison, S. J. (2007). Physiological Notch signaling promotes gliogenesis in the developing peripheral and central nervous systems. Development 134, 2435-2447. doi: 10.1242/dev.005520

Thompson, C. L., Ng, L., Menon, V., Martinez, S., Lee, C.-K., Glattfelder, K., et al. (2014). A high-resolution spatiotemporal atlas of gene expression of the developing mouse brain. Neuron 83, 309-323. doi: 10.1016/j.neuron.2014.05.033

Wang, J., Duncan, D., Shi, Z., and Zhang, B. (2013). WEB-based GEne SeT AnaLysis Toolkit (WebGestalt): update 2013. Nucleic Acids Res. 41, W77-W83. doi: 10.1093/nar/gkt439

Wang, S., Sdrulla, A. D., DiSibio, G., Bush, G., Nofziger, D., Hicks, C., et al. (1998). Notch receptor activation inhibits oligodendrocyte differentiation. Neuron 21, 63-75. doi: 10.1016/S0896-6273(00)80515-2

Wen, J., Hu, Q., Li, M., Wang, S., Zhang, L., Chen, Y., et al. (2008). Pax6 directly modulate Sox2 expression in the neural progenitor cells. Neuroreport 19, 413-417. doi: 10.1097/WNR.0b013e3282f64377

Wilkinson, G., Dennis, D., and Schuurmans, C. (2013). Proneural genes in neocortical development. Neuroscience 253, 256-273. doi: 10.1016/j.neuroscience.2013.08.029

Wirtz-Peitz, F., Nishimura, T., and Knoblich, J. A. (2008). Linking cell cycle to asymmetric division: aurora-A phosphorylates the Par complex to regulate Numb localization. Cell 135, 161-173. doi: 10.1016/j.cell.2008.07.049

Xia, Y.-P., Dai, R.-L., Li, Y.-N., Mao, L., Xue, Y.-M., He, Q.-W., et al. (2012). The protective effect of sonic hedgehog is mediated by the phosphoinositide 3-kinase/AKT/Bcl-2 pathway in cultured rat astrocytes under oxidative stress. Neuroscience 209, 1-11. doi: 10.1016/j.neuroscience.2012.02.019

Yang, X., Klein, R., Tian, X., Cheng, H.-T., Kopan, R., and Shen, J. (2004). Notch activation induces apoptosis in neural progenitor cells through a p53-dependent pathway. Dev. Biol. 269, 81-94. doi: 10.1016/j.ydbio.2004.01.014

Yoon, K., and Gaiano, N. (2005). Notch signaling in the mammalian central nervous system: insights from mouse mutants. Nat. Neurosci. 8, 709-715. doi: $10.1038 / \mathrm{nn} 1475$

Yoshikawa, K. (2000). Cell cycle regulators in neural stem cells and postmitotic neurons. Neurosci. Res. 37, 1-14. doi: 10.1016/S0168-0102(00)00101-2
Yu, W., McDonnell, K., Taketo, M. M., and Bai, C. B. (2008). Wnt signaling determines ventral spinal cord cell fates in a time-dependent manner. Development 135, 3687-3696. doi: 10.1242/dev.021899

Zhang, B., Kirov, S., and Snoddy, J. (2005). WebGestalt: an integrated system for exploring gene sets in various biological contexts. Nucleic Acids Res. 33, W741-W748. doi: 10.1093/nar/gki475

Zhang, H., Deo, M., Thompson, R. C., Uhler, M. D., and Turner, D. L. (2012a). Negative regulation of Yap during neuronal differentiation. Dev. Biol. 361, 103-115. doi: 10.1016/j.ydbio.2011.10.017

Zhang, J., Shemezis, J. R., McQuinn, E. R., Wang, J., Sverdlov, M., and Chenn, A. (2013). AKT activation by $\mathrm{N}$-cadherin regulates beta-catenin signaling and neuronal differentiation during cortical development. Neural Dev. 8, 7. doi: 10.1186/1749-8104-8-7

Zhang, W., Kang, J.-S., Cole, F., Yi, M.-J., and Krauss, R. S. (2006). Cdo functions at multiple points in the Sonic Hedgehog pathway, and Cdo-deficient mice accurately model human holoprosencephaly. Dev. Cell 10, 657-665. doi: 10.1016/j.devcel.2006.04.005

Zhang, X., Chen, T., Zhang, J., Mao, Q., Li, S., Xiong, W., et al. (2012b). Notch1 promotes glioma cell migration and invasion by stimulating $\beta$-catenin and NF$\mathrm{B}$ signaling via AKT activation. Cancer Sci. 103, 181-190. doi: 10.1111/j.13497006.2011.02154.x

Zhao, L., Zevallos, S. E., Rizzoti, K., Jeong, Y., Lovell-Badge, R., and Epstein, D. J. (2012). Disruption of SoxB1-dependent Sonic hedgehog expression in the hypothalamus causes septo-optic dysplasia. Dev. Cell 22, 585-596. doi: 10.1016/j.devcel.2011.12.023

Zhao, T., Gan, Q., Stokes, A., Lassiter, R. N. T., Wang, Y., Chan, J., et al. (2014). $\beta$ catenin regulates Pax3 and $\mathrm{Cdx} 2$ for caudal neural tube closure and elongation. Development 141, 148-157. doi: 10.1242/dev.101550

Zuberi, K., Franz, M., Rodriguez, H., Montojo, J., Lopes, C. T., Bader, G. D., et al. (2013). GeneMANIA prediction server 2013 update. Nucleic Acids Res. 41, W115-W122. doi: 10.1093/nar/gkt533

Conflict of Interest Statement: The authors declare that the research was conducted in the absence of any commercial or financial relationships that could be construed as a potential conflict of interest.

Received: 30 June 2014; accepted: 01 August 2014; published online: 21 August 2014. Citation: Vied CM, Freudenberg F, Wang Y, Raposo AASF, Feng D and Nowakowski RS (2014) A multi-resource data integration approach: identification of candidate genes regulating cell proliferation during neocortical development. Front. Neurosci. 8:257. doi: 10.3389/fnins.2014.00257

This article was submitted to Neurogenomics, a section of the journal Frontiers in Neuroscience.

Copyright (C) 2014 Vied, Freudenberg, Wang, Raposo, Feng and Nowakowski. This is an open-access article distributed under the terms of the Creative Commons Attribution License (CC BY). The use, distribution or reproduction in other forums is permitted, provided the original author(s) or licensor are credited and that the original publication in this journal is cited, in accordance with accepted academic practice. No use, distribution or reproduction is permitted which does not comply with these terms. 